\title{
On the Zeros of Blocked Linear Systems with Single and Mixed Frequency Data
}

\author{
Mohsen Zamani, Wetian Chen, Senior Member, IEEE, Brian D.O. Anderson, Life Fellow, IEEE, \\ Manfred Deistler, Fellow, IEEE and Alexander Filler.
}

\begin{abstract}
This paper studies properties of blocked systems resulting from blocking discrete linear systems with mixed frequency data. The focus is on the zeros of the blocked systems. We first establish results on the simpler single frequency case, where the unblocked linear systems have all data at the same frequency. In particular, an explicit relation between the system matrix of the unblocked linear systems and that of their corresponding blocked systems is derived. Based on this relation, it is shown that the blocked systems are zero free if and only if the related unblocked systems are zero free. Furthermore, it is illustrated that square systems have zeros generically, i.e. for generic parameter matrices, and the corresponding kernel is of dimension one. With the help of the results obtained for the single frequency case, we then identify a situation in which the blocked systems can be zero free.
\end{abstract}

\section{INTRODUCTION}

In different branches of science and technology, one has to deal with dynamic systems with mixed frequency data. For instance, in econometric modeling, it is common to have data which are collected monthly, while some other data may be obtained quarterly or even annually [1]. In signal processing and systems and control, mixed frequency data arises naturally from multi-rate sampled data systems [2], [3].

In order to deal with systems which have mixed frequency data, a technique called blocking or lifting has been developed in signal processing and systems and control [3]. This method was introduced to transform linear discretetime periodic systems into linear time-invariant systems so that the well-established analysis and design tools in linear time-invariant systems can be extended to linear discretetime periodic systems [4], [5], [6] and [7] . For example, the authors in [4] and [5] extended the notions of poles and zeros of linear time-invariant systems to linear periodic systems. Some necessary and sufficient conditions for structural properties such as observability and reachability have been studied in [8] and [9]. Moreover, the realization problem has been researched in [9] and the related references listed therein. The blocking technique can also be performed on time-invariant systems as well. For instance, in [10] linear

M. Zamani, W. Chen and B. D. O. Anderson are with Research School of Information Sciences and Engineering, Australian National University, Canberra, ACT 0200, Australia. B. D. O. Anderson is with National ICT Australia (NICTA). \{mohsen.zamani, weitian.chen, brian. anderson\} @anu.edu. au

M. Deistler is with Department of mathematical methods in Economics, Technical university of Vienna, 8/119 Argentinierstrasse, A 1040 Vienna, Austria. deistlerduwien.ac.at

A. Filler is with Wagner, Elbling and Company, Annagasse 5/3/14, A1010 Vienna, Austria. alexfilleregmx.at time-invariant discrete-time systems have been blocked for designing periodic controllers. In [11], for linear systems with single frequency data, the authors obtained several interesting results on the zeros of the blocked systems by using the tool of matrix fraction description (MFD).

In this paper, we assume there exists an underlying high frequency system, which is linear and time-invariant. However, because not all the outputs of this underlying system are measured at the same frequency, we end up with a linear system with mixed frequency data. Linear systems with mixed frequency data are actually a very special class of linear periodic systems. For this special class of systems, little is known about the zero properties of their blocked systems. For example, it is not clear whether the blocked systems have zeros or not. Moreover, when the blocked systems do have zeros, it is not known yet how the zeros of the blocked system are related to the zeros of the underlying linear time-invariant system.

Furthermore, when one is considering the generic setting i.e. for almost all choices of parameter matrices of a minimal state space system, there is no result about the nullity of the system matrix of the blocked system. The main objective of this paper is to focus on the zero properties of the blocked systems of linear systems with mixed frequency data and provide answers to the problems raised in the previous paragraph. To achieve this goal, we start by studying the zero properties of the blocked systems resulting from blocking linear systems with single frequency data (This case has been dealt with in [11] using the MFD approach, but here we use a time domain approach which can provide a more explicit and insightful relation). Besides being much simpler, the results obtained for the single frequency data case are essential for the results in the mixed frequency data case. The main idea is to first derive a relation between the system matrix of the blocked systems and that of the unblocked linear time-invariant systems. Then based on this relation, we can obtain results about the zeros of the blocked systems for the single frequency data case. Finally, we can establish the zero properties of the blocked systems in the mixed frequency data case.

The structure of the paper is as follows. Section II establishes the relation between zeros of blocked systems and zeros of the linear time-invariant unblocked systems for single frequency data case and presents some interesting results. In Section III, systems with mixed frequency data are studied. Finally, Section IV concludes. 


\section{LINEAR SYSTEMS WITH SINGLE FREQUENCY DATA}

The time-invariant unblocked system under consideration is described as

$$
\begin{aligned}
x(k+1) & =A x(k)+B u(k) \\
y(k) & =C x(k)+D u(k)
\end{aligned}
$$

where $k \in \mathbb{Z}, x(k) \in \mathbb{R}^{n}, y(k) \in \mathbb{R}^{p}$ and $u(k) \in \mathbb{R}^{m}$, $p \geq m$. For this system, we assume that $y(k)$ is available at every time instant $k$. For consistency of nomenclature, we call this case a single frequency data case.

Define

$$
\begin{aligned}
v(k) & =\left[\begin{array}{llll}
u^{T}(k) & u^{T}(k+1) & \ldots & u^{T}(k+N-1)
\end{array}\right]^{T}, \\
q(k) & =\left[\begin{array}{llll}
y^{T}(k) & y^{T}(k+1) & \ldots & y^{T}(k+N-1)
\end{array}\right]^{T},
\end{aligned}
$$

where $k=0, N, 2 N, \ldots$.

Then the blocked system is given by

$$
\begin{aligned}
x(k+N) & =A_{b} x(k)+B_{b} v(k) \\
q(k) & =C_{b} x(k)+D_{b} v(k),
\end{aligned}
$$

where

$$
\begin{aligned}
A_{b}= & A^{N}, \quad B_{b}=\left[\begin{array}{llll}
A^{N-1} B & A^{N-2} B & \ldots & B
\end{array}\right], \\
C_{b}= & {\left[\begin{array}{ccccc}
C^{T} & A^{T} C^{T} & \ldots & A^{(N-1)^{T}} C^{T}
\end{array}\right]^{T}, } \\
D_{b}= & {\left[\begin{array}{cccc}
D & 0 & \ldots & 0 \\
C B & D & \ldots & 0 \\
\vdots & \vdots & \ddots & \ldots \\
C A^{N-2} B & C A^{N-3} B & \ldots & D
\end{array}\right] }
\end{aligned}
$$

An operator $Z$ is defined such that $Z x(k)=x(k+N)$, $Z v(k)=v(k+N), Z q(k)=q(k+N)$. The symbol $Z$ is also used to denote a complex value.

As we focus on the properties of zeros in this paper, below we define zeros for systems (1) and (3).

Definition 2.1: The finite zeros of the transfer function $W(z)=C(z I-A)^{-1} B+D$ with minimal realization $\{A, B, C, D\}$ are defined to be the finite values of $z$ for which the rank of the following system matrix falls below its normal rank

$$
M(z)=\left[\begin{array}{cc}
z I-A & -B \\
C & D
\end{array}\right] .
$$

Further, $W(z)$ is said to have an infinite zero when $n+$ $\operatorname{rank}(D)$ is less than the normal rank of $M(z)$, or equivalently the rank of $D$ is less than the normal rank of $W(z)$.

Definition 2.2: The finite zeros of the transfer function $V(Z)=C_{b}\left(Z I-A_{b}\right)^{-1} B_{b}+D_{b}$ with minimal realization $\left\{A_{b}, B_{b}, C_{b}, D_{b}\right\}$ are defined to be the finite values of $Z$ for which the rank of the following system matrix falls below its normal rank

$$
M_{b}(Z)=\left[\begin{array}{cc}
Z I-A_{b} & -B_{b} \\
C_{b} & D_{b}
\end{array}\right] .
$$

Further, $V(Z)$ is said to have an infinite zero when $n+$ $\operatorname{rank}\left(D_{b}\right)$ is less than the normal rank of $M_{b}(Z)$, or equivalently the rank of $D_{b}$ is less than the normal rank of $V(Z)$.

We are interested in studying the relation between zeros of (3) and (1). For any $z_{0}$ and $Z_{0}$ such that $Z_{0}=z_{0}^{N}$, we are going to investigate the relation between $M_{b}\left(Z_{0}\right)$ and $M\left(z_{0}\right)$ which will in turn reveal the relation between the zeros of (1) and (3).

Lemma 2.3: For any matrix polynomial $P(S)=S^{N} I-$ $A^{N}$, where $S \in \mathbb{C}, A \in \mathbb{R}^{n \times n}$ and $I$ an identity matrix with proper dimension and $N \in \mathbb{N}$ the following identity holds

$$
\begin{aligned}
P(S)=S^{N} I-A^{N}= & (-1)^{N+1}(S I-A)(\omega S I-A) \\
& \left(\omega^{2} S I-A\right) \ldots\left(\omega^{N-1} S I-A\right),
\end{aligned}
$$

where $\omega \in \mathbb{C}, \omega=\exp \left(\frac{2 \pi j}{N}\right)$ and $j=\sqrt{-1}$.

The above lemma will enable us to decompose the system matrix $M_{b}\left(Z_{0}\right)$ into a product of several well-structured matrices as shown in the next example.

Example 2.4: To illustrate the relation between $M_{b}\left(Z_{0}\right)$ and $M\left(z_{0}\right)$, the case $N=3$ is shown. Let $z_{0}$ be defined as any cube root of $Z_{0}$. Then one can write

$$
\begin{aligned}
& {\left[\begin{array}{ccc}
Z_{0} I-A_{b} & -B_{b} \\
C_{b} & D_{b}
\end{array}\right]=} \\
& {\left[\begin{array}{cccc}
z_{0}^{3} I-A^{3} & -A^{2} B & -A B & -B \\
C & D & 0 & 0 \\
C A & C B & D & 0 \\
C A^{2} & C A B & C B & D
\end{array}\right]=} \\
& {\left[\begin{array}{cccc}
\omega^{2} z_{0} I-A & 0 & 0 & -B \\
0 & -I_{p} & 0 & 0 \\
0 & \omega^{2} z_{0} I_{p} & -I_{p} & 0 \\
C & 0 & \omega^{2} z_{0} I_{p} & D
\end{array}\right]} \\
& {\left[\begin{array}{cccc}
\omega z_{0} I-A & 0 & -B & 0 \\
0 & -I_{p} & 0 & 0 \\
C & \omega z_{0} I_{p} & D & 0 \\
0 & 0 & \omega z_{0} I_{m} & -I_{m}
\end{array}\right]} \\
& {\left[\begin{array}{cccc}
z_{0} I-A & -B & 0 & 0 \\
C & D & 0 & 0 \\
0 & z_{0} I_{m} & -I_{m} & 0 \\
0 & 0 & z_{0} I_{m} & -I_{m}
\end{array}\right]}
\end{aligned}
$$

where $\omega=\exp \left(\frac{2 \pi j}{3}\right)$.

Example 2.5: For further explanation the case $N=4$ is shown in this example. $z_{0}$ is defined by $Z_{0}=z_{0}^{4}$. One can write

$$
\begin{aligned}
& {\left[\begin{array}{cc}
Z_{0} I-A_{b} & -B_{b} \\
C_{b} & D_{b}
\end{array}\right]=} \\
& {\left[\begin{array}{ccccc}
z_{0}^{4} I-A^{4} & -A^{3} B & -A^{2} B & -A B & -B \\
C & D & 0 & 0 & 0 \\
C A & C B & D & 0 & 0 \\
C A^{2} & C A B & C B & D & 0 \\
C A^{3} & C A^{2} B & C A B & C B & D
\end{array}\right]=}
\end{aligned}
$$




$$
\begin{gathered}
{\left[\begin{array}{ccccc}
\omega z_{0} I-A & 0 & 0 & 0 & -B \\
0 & -I_{p} & 0 & 0 & 0 \\
0 & \omega z_{0} I_{p} & -I_{p} & 0 & 0 \\
0 & 0 & \omega z_{0} I_{p} & -I_{p} & 0 \\
C & 0 & 0 & \omega z_{0} I_{p} & D
\end{array}\right]} \\
{\left[\begin{array}{ccccc}
\omega^{3} z_{0} I-A & 0 & 0 & -B & 0 \\
0 & -I_{p} & 0 & 0 & 0 \\
0 & \omega^{3} z_{0} I_{p} & -I_{p} & 0 & 0 \\
C & 0 & \omega^{3} z_{0} I_{p} & D & 0 \\
0 & 0 & 0 & \omega^{3} z_{0} I_{m} & -I_{m}
\end{array}\right]} \\
{\left[\begin{array}{ccccc}
z_{0} I-A & 0 & -B & 0 & 0 \\
0 & -I_{p} & 0 & 0 & 0 \\
C & z_{0} I_{m} & D & 0 & 0 \\
0 & 0 & z_{0} I_{m} & -I_{m} & 0 \\
0 & 0 & 0 & z_{0} I_{m} & -I_{m}
\end{array}\right]} \\
{\left[\begin{array}{ccccc}
\omega^{2} z_{0} I-A & -B & 0 & 0 & \\
C & D & 0 & 0 & 0 \\
0 & \omega^{2} z_{0} I_{m} & -I_{m} & 0 & 0 \\
0 & 0 & \omega^{2} z_{0} I_{m} & -I_{m} & 0 \\
0 & 0 & 0 & \omega^{2} z_{0} I_{m} & -I_{m}
\end{array}\right],}
\end{gathered}
$$

$$
\text { where } \omega=\exp \left(\frac{2 \pi j}{4}\right) \text {. }
$$

The decompositions in the previous examples turn out to be generalizable to any $N$. For the sake of simplicity, we just focus on the case where $N$ is odd. The system matrix of (3) can be written as

$$
\begin{aligned}
& M_{b}\left(Z_{0}\right)=\left[\begin{array}{cc}
Z_{0} I-A_{b} & -B_{b} \\
C_{b} & D_{b}
\end{array}\right]= \\
& {\left[\begin{array}{ccccc}
z_{0}^{N} I-A^{N} & -A^{N-1} B & -A^{N-2} B & \ldots & -B \\
C & D & 0 & & 0 \\
C A & C B & D & & \\
\vdots & \vdots & \vdots & & \vdots \\
C A^{N-1} & C A^{N-2} B & C A^{N-3} B & & D
\end{array}\right],}
\end{aligned}
$$

where $z_{0}$ can be any $N$-th root of $Z_{0}$. Via simple algebra it can be checked that the following relation holds

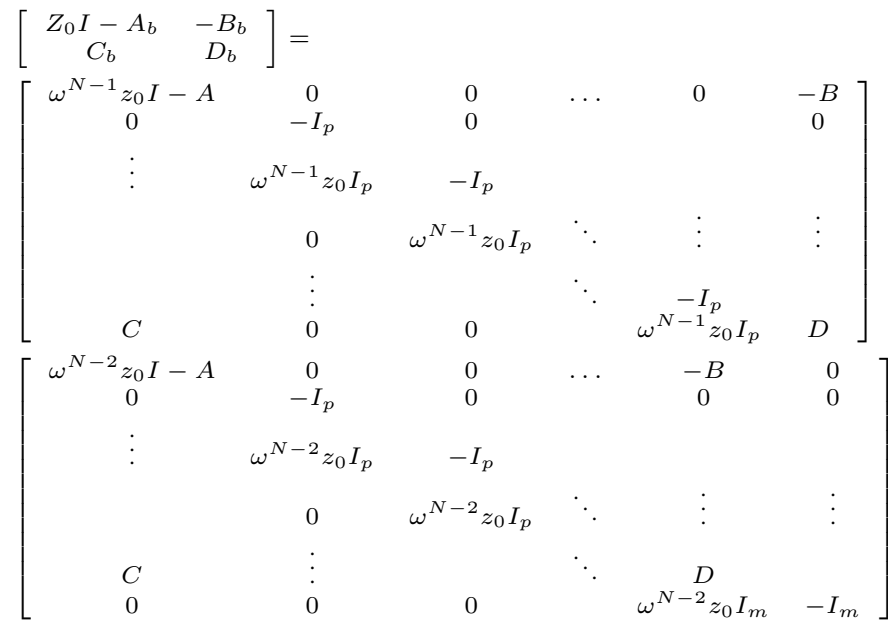

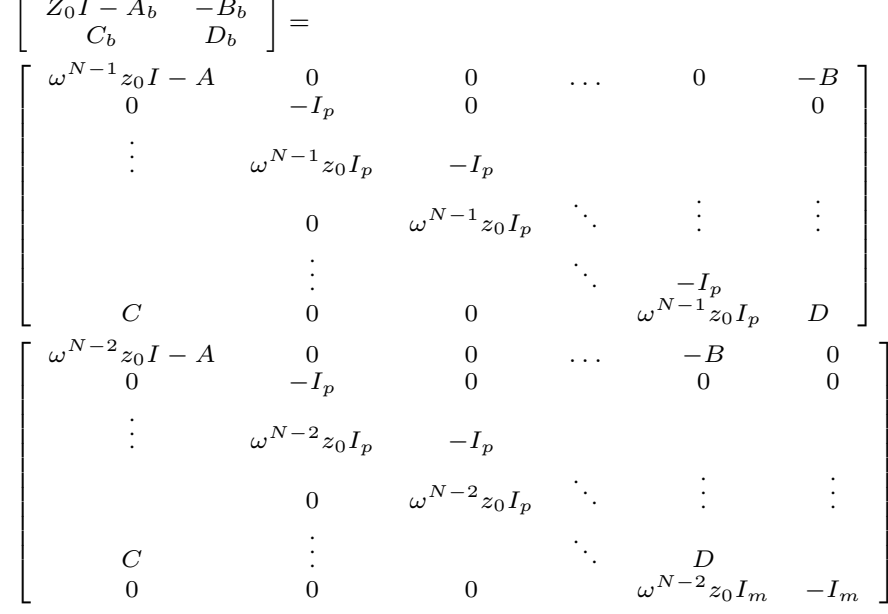

$$
\cdots *\left[\begin{array}{ccccccc}
\omega z_{0} I-A & 0 & -B & & & 0 & 0 \\
0 & -I_{p} & 0 & \ldots & & 0 & 0 \\
C & \omega z_{0} I_{p} & D & & & & 0 \\
0 & 0 & \omega z_{0} I_{m} & -I_{m} & 0 & \ldots & \\
& 0 & 0 & & & \vdots & \vdots \\
\vdots & \vdots & 0 & \ddots & \ddots & & \\
& & \vdots & & \ddots & -I_{m} & \\
0 & 0 & 0 & & & \omega z_{0} I_{m} & -I_{m}
\end{array}\right]
$$$$
\left[\begin{array}{ccccccc}
z_{0} I-A & -B & 0 & & \cdots & & 0 \\
C & D & 0 & & & 0 & \\
0 & z_{0} I_{m} & -I_{m} & 0 & & \vdots & \\
& 0 & z_{0} I_{m} & -I_{m} & & & \vdots \\
\vdots & \vdots & 0 & z_{0} I_{m} & -I_{m} & & \\
\vdots & \vdots & \vdots & \vdots & & -I_{m} & \\
0 & 0 & 0 & 0 & & z_{0} I_{m} & -I_{m}
\end{array}\right] \text { (10) }
$$

where $\omega$ can be defined as $\omega=\exp \left(\frac{2 \pi j}{N}\right)$.

The above equation clearly establishes a relation between the system matrix $M_{b}\left(Z_{0}\right)$ of (3) and the system matrices $M\left(\omega^{N-1} z_{0}\right), M\left(\omega^{N-2} z_{0}\right), \ldots, M\left(z_{0}\right)$ as defined by (1) which appear as principal block submatrices of the product terms on the right of (10).

Based on (10), the following results can be obtained which make a linkage between zeros of the system (3) and zeros of the system (1).

Lemma 2.6: For a generic choice of matrices $\{A, B, C, D\}$ with $p \geq m$, the system matrix of (1) has normal rank equal to $n+m$.

Proof: Observe that

$$
\begin{aligned}
& M(z)=\left[\begin{array}{cc}
z I-A & -B \\
C & D
\end{array}\right]= \\
& {\left[\begin{array}{cc}
I & 0 \\
C(z I-A)^{-1} & I
\end{array}\right]\left[\begin{array}{cc}
z I-A & -B \\
0 & C(z I-A)^{-1} B+D
\end{array}\right] .}
\end{aligned}
$$

Consider that $M(z)$ has $n+m$ columns so, $n+m \geq$ normal $\operatorname{rank}(M(z))=$ normal $\operatorname{rank}(z I-A)+$ normal $\operatorname{rank}(C(z I-$ $\left.A)^{-1} B+D\right) \geq n+\operatorname{rank}\left(\lim _{z \rightarrow \infty}\left[C(z I-A)^{-1} B+D\right]\right)=n+$ $\operatorname{rank}(D)=n+m$. Hence, the normal rank of $M(z)$ equals the number of its columns.

Lemma 2.7: For a generic choice of matrices $\{A, B, C, D\}$ with $p \geq m$, the system matrix of (3) has normal rank equal to $n+N m$.

Proof: In the generic setting and $p \geq m$, matrix $D$ is of full column rank. So, due to the structure of $D_{b}$, see (4), one can easily conclude that $D_{b}$ is of full column rank as well. Thus, the proof can be done in the similar way as the proof of the previous lemma.

Lemma 2.8: Suppose that $p \geq m$. Then the normal rank of $M(z)$ is $n+m$ if and only if the normal rank $M_{b}(Z)$ is $n+N m$.

\section{Proof:}

Sufficiency: If $M_{b}(Z)$ has normal rank of $n+N m$, then due to the structure of (10), it is immediate that $M(z)$ must 
have full-column normal rank.

Necessity: If $M_{b}(Z)$ has normal rank less than $n+N m$ then based on the decomposition (10), one or more of the system matrices $M\left(\omega^{N-1} z\right), M\left(\omega^{N-2} z\right), \ldots, M(z)$ as defined by (1) which appear as principal block submatrices of the product terms on the right of (10) cannot attain fullcolumn normal rank.

Theorem 2.9: Suppose the system matrix of (1) has fullcolumn normal rank. Then if (1) has a finite zero at $z=z_{0} \neq$ 0 , then the system (3) has a finite zero at $Z=Z_{0}=z_{0}^{N} \neq 0$.

Proof: Assume that the system (1) has a finite zero at $z_{0}$, then there exists a nonzero vector

$$
\gamma=\left[\begin{array}{ll}
x_{0}^{T} & u_{0}^{T}
\end{array}\right]^{T},
$$

such that

$$
\left[\begin{array}{cc}
z_{0} I-A & -B \\
C & D
\end{array}\right]\left[\begin{array}{l}
x_{0} \\
u_{0}
\end{array}\right]=0 .
$$

Then it readily follows from (10) that there exists a nonzero vector

$$
\Upsilon=\left[\begin{array}{llllll}
x_{0}^{T} & u_{0}^{T} & z_{0} u_{0}^{T} & z_{0}^{2} u_{0}^{T} & \ldots & z_{0}^{N-1} u_{0}^{T}
\end{array}\right]^{T},
$$

such that

$$
\left[\begin{array}{cc}
Z_{0} I-A_{b} & -B_{b} \\
C_{b} & D_{b}
\end{array}\right] \Upsilon=0 .
$$

This implies that the system matrix of (3) has less than full column rank. Based on the assumption $M(z)$ has full-column normal rank. Hence, due to the conclusion of Lemma 2.8, the normal rank for system matrix $M_{b}(Z)$ equals the number of columns. Thus, (3) must have a zero at $Z_{0}$. This ends the proof.

Theorem 2.10: Suppose the system matrix of (1) has fullcolumn normal rank. Then if the system (3) has a finite zero at $Z=Z_{0}=z_{0}^{N} \neq 0$, then the system (1) has a finite zero at one or more of $z=z_{0} \neq 0$ or $z=\omega z_{0} \neq 0 \ldots$ $z=\omega^{N-1} z_{0} \neq 0$, where $\omega=\exp \left(\frac{2 \pi j}{N}\right)$.

Proof: Assume that the system (3) has a zero at $Z_{0}$, then there exists a nonzero vector

$$
\bar{\Upsilon}=\left[\begin{array}{lllll}
\bar{x}_{0}^{T} & \bar{u}_{0}^{T} & \bar{u}_{1}^{T} & \ldots & \bar{u}_{N-1}^{T}
\end{array}\right]^{T},
$$

such that

$$
\left[\begin{array}{cc}
Z_{0} I-A_{b} & -B_{b} \\
C_{b} & D_{b}
\end{array}\right] \bar{\Upsilon}=0 .
$$

Without loss of generality we focus on (10), where there must exist at least one matrix in the right hand side of (10) which has less than full column rank. This implies that one or more of the system matrices $M\left(\omega^{N-1} z_{0}\right), M\left(\omega^{N-2} z_{0}\right), \ldots, M\left(z_{0}\right)$ as defined by (1) which appear as principal block submatrices of the product terms on the right of (10) has less than full column rank. Moreover, the conclusion of Lemma 2.8 implies that system matrix $M(z)$ has normal rank equal to full column rank. Hence, the system (1) has zero at one or more of $z=z_{0} \neq 0$ or $z=\omega z_{0} \neq 0 \ldots z=\omega^{N-1} z_{0} \neq 0$, where $\omega=\exp \left(\frac{2 \pi j}{N}\right)$.

Theorem 2.11: Suppose the system matrix of (1) has fullcolumn normal rank. Then system (3) has a zero at $Z_{0}=\infty$ if and only if the system (1) has a zero at $z_{0}=\infty$.

Proof:

According to the definition of zero at infinity and assumption provided in statement of theorem, $W(z)$ has a zero at infinity if and only if $\operatorname{rank}(D)<m$. Moreover, $V(Z)$ has zero at $Z=\infty$ if and only if $\operatorname{rank}\left(D_{b}\right)<m N$.

If $W(z)$ has a zero at $z_{0}=\infty$, then there exists a nonzero vector

$$
\widehat{\gamma}=\widehat{u}_{0}
$$

such that

$$
D \widehat{\gamma}=0
$$

Since

$$
D_{b}=\left[\begin{array}{cccc}
D & 0 & \ldots & 0 \\
C B & D & \ldots & 0 \\
\vdots & \vdots & \ddots & \ldots \\
C A^{N-2} B & C A^{N-3} B & \ldots & D
\end{array}\right],
$$

then there exists a vector

$$
\widehat{\Upsilon}=\left[\begin{array}{lllll}
0 & 0 & 0 & \ldots & \widehat{u}_{0}^{T}
\end{array}\right]^{T} .
$$

Such that $D_{b} \widehat{\Upsilon}=0$. Conversely, it can be easily shown that if $\operatorname{rank}\left(D_{b}\right)<m N$, then $\operatorname{rank}(D)<m$.

Theorem 2.12: Suppose the system matrix of (1) has fullcolumn normal rank. Then the system (3) has a zero at $Z_{0}=$ 0 if and only if the system (1) has a zero at $z_{0}=0$.

Proof:

Sufficiency: Suppose the system (1) has a zero at $z_{0}=0$, then there exists a nonzero vector

$$
\tilde{\gamma}=\left[\begin{array}{c}
\tilde{x}_{0} \\
\tilde{u}_{0}
\end{array}\right]
$$

such that

$$
\left[\begin{array}{cc}
-A & B \\
C & D
\end{array}\right]\left[\begin{array}{l}
\tilde{x}_{0} \\
\tilde{u}_{0}
\end{array}\right]=0
$$

We now show that the system (3) has a zero at $Z_{0}=0$. We have

$$
\begin{aligned}
& {\left[\begin{array}{cc}
-A_{b} & -B_{b} \\
C_{b} & D_{b}
\end{array}\right]=} \\
& {\left[\begin{array}{ccccccc}
-A & 0 & 0 & & & 0 & -B \\
0 & -I_{p} & 0 & & & & 0 \\
0 & 0 & -I_{p} & & & & \\
0 & 0 & 0 & \ddots & & \vdots & \vdots \\
& \vdots & 0 & \ddots & \ddots & & \\
\vdots & & \vdots & & \ddots & -I_{p} & \\
C & 0 & 0 & & & 0 & D
\end{array}\right]}
\end{aligned}
$$




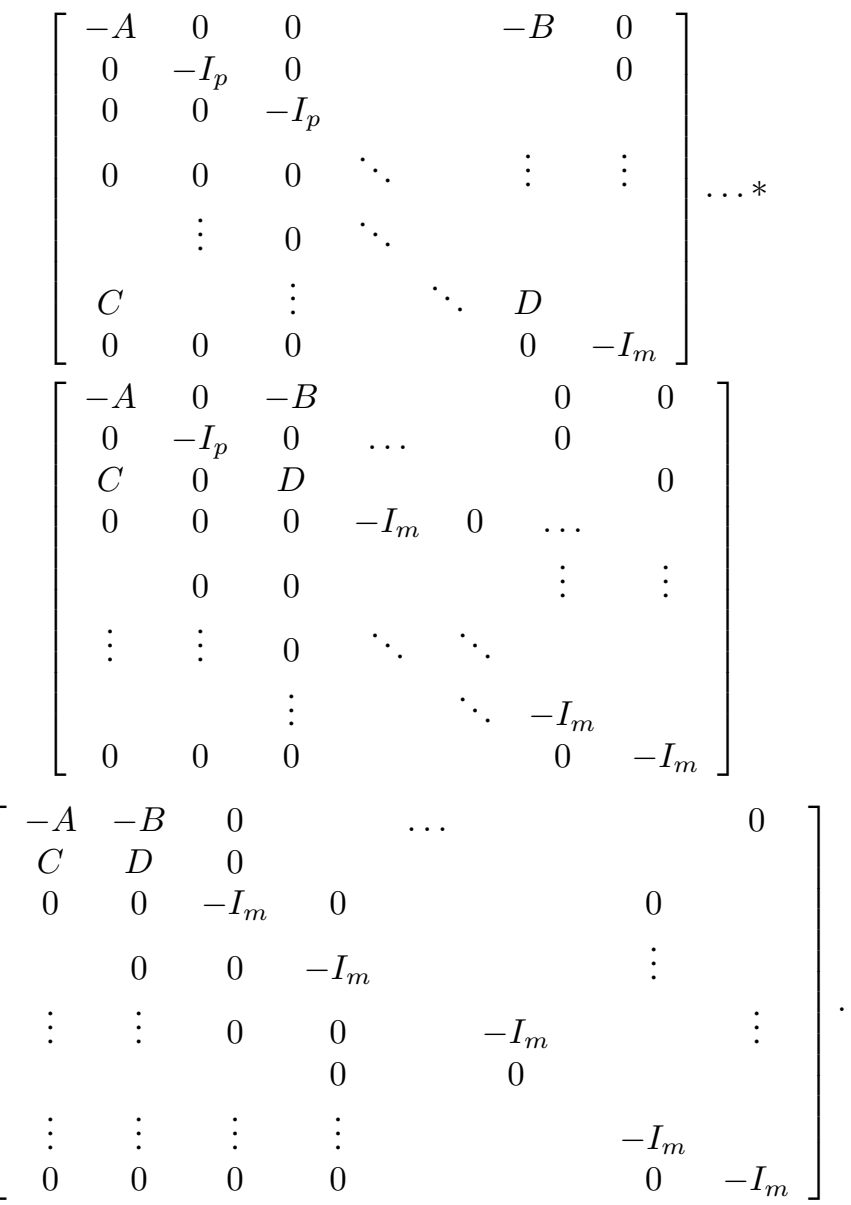

Assume now with

$$
\tilde{\Upsilon}=\left[\begin{array}{lllll}
\tilde{x}_{0}^{T} & \tilde{u}_{0}^{T} & 0 & \ldots & 0
\end{array}\right]^{T},
$$

there follows

$$
\left[\begin{array}{cc}
-A_{b} & -B_{b} \\
C_{b} & D_{b}
\end{array}\right] \tilde{\Upsilon}=0
$$

Necessity: Assume that (3) has a zero at $Z_{0}=0$, then there must exist at least one matrix in the right hand side of (11) which is less than full column rank. This implies that system (1) has a zero at $z_{0}=0$.

Theorem 2.13: Consider the system (1) defined by the quadruple $\{A, B, C, D\}$, in which the individual matrices are generic. Then

1) If $p>m$, the system matrix of the blocked system has full column rank for all $z$.

2) If $p=m$, then the system matrix of the blocked system can only have finite zeros with one-dimensional kernel. Proof:

Suppose first that $p>m$. It was shown in Lemma 2.6 that the system matrix of the generic unblocked system has full-column normal rank. Furthermore, [12] showed that for the generic systems, the system matrix has rank equal to its normal rank for all $z$. If the blocked system had a system matrix with less than full column rank for a finite $Z_{0} \neq 0$, then according to Theorem 2.10, there would be necessarily a nonzero nullvector of the system matrix of the unblocked system for $z_{0} \neq 0$ equal to some $N-t h$ root of $Z_{0}$, which would be a contradiction. If the blocked system had a zero at $Z_{0}=\infty$, then based on Theorem 2.11 the $D$ matrix of the unblocked system would be less than full column rank which would be a contradiction. Analogously, using the argument in Theorem 2.12, one can easily conclude that blocked system has full column rank system matrix at $Z_{0}=0$.

Now we consider the case $p=m$; since $D$ is generic, it has full column rank. Hence, based on the conclusion of Theorem 2.11, both the unblocked system and the blocked system do not have zeros at infinity. In the second part of this proof we use the conclusion of Theorem 2.9. Moreover, based on the fact that $D_{b}$ is nonsingular, one can conclude that the zeros of the blocked system are the eigenvalues of $A_{b}-B_{b} D_{b}^{-1} C_{b}$. If the eigenvalues of $A_{b}-B_{b} D_{b}^{-1} C_{b}$ are distinct, then the associated eigenspace for each eigenvalue is one-dimensional; it is equivalent to say that the associated kernel for each zero of $M_{b}(Z)$ has dimension one. One should note that the unblocked system has distinct zeros due to the genericity assumption. Furthermore, zeros of the unblocked system generically have distinct magnitudes except for complex conjugate pairs. It is obvious that those zeros of the unblocked system with distinct magnitudes produce distinct blocked zeros. Now, we focus on zeros of the unblocked system with the same magnitudes, i.e. complex conjugate pairs. The only case where the generic unblocked system has distinct zeros but its corresponding blocked system has nondistinct zeros happens when the $N-t h$ power of the complex conjugate zeros of the unblocked system coincide. We now show by contradiction that this is generically impossible. In order to illustrate a contradiction, suppose that the unblocked system has a complex conjugate pair, say $z_{01}$ and $z_{01}^{*}$. If they produce an identical zero for the blocked system, their $N-t h$ power must be the same. The latter condition implies that the angle between $z_{01}$ and $z_{01}^{*}$ has to be exactly $\frac{2 \pi h}{N}$, where $h$ is an integer, which contradicts the genericity assumption for the unblocked system. Hence, the zeros of the blocked system have distinct values and consequently the corresponding kernels of system matrix are one-dimensional.

\section{LINEAR SYSTEMS WITH MIXED FREQUENCY DATA}

In this section, we focus on linear systems with mixed frequency data. We use the results obtained in the previous section to study the zero properties of the blocked systems resulting from blocking of linear systems with mixed frequency data. In this paper, we only study a case where there exist systems with two time scale data. However, the obtained results can be readily extended to a general system with more than two time scale data.

For systems with two time scale data, we can decompose the output $y(k)$ as

$$
y(k)=\left[\begin{array}{ll}
y^{f}(k)^{T} & y^{s}(k)^{T}
\end{array}\right]^{T},
$$

where $y^{f}(k) \in \mathbb{R}^{p_{1}}$ and $y^{s}(k) \in \mathbb{R}^{p_{2}}, p_{1}+p_{2}=p$, represent high frequency data and low frequency data, accordingly. 
More specifically, $y^{f}(k)$ is observed at all time instant $k=0,1,2, \ldots$, while $y^{s}(k)$ is observed at $0, N, 2 N, \ldots$. Consequently, we can decompose $C$ and $D$ as

$$
\begin{aligned}
& C=\left[\begin{array}{ll}
C^{f^{T}} & C^{s^{T}}
\end{array}\right]^{T}, \\
& D=\left[\begin{array}{ll}
D^{f^{T}} & D^{s^{T}}
\end{array}\right]^{T} .
\end{aligned}
$$

Define

$$
\begin{aligned}
U(k)= & {\left[\begin{array}{c}
u(k) \\
u(k+1) \\
\cdots \\
u(k+N-1)
\end{array}\right], } \\
Y(k)= & {\left[\begin{array}{c}
y^{f}(k) \\
y^{f}(k+1) \\
\vdots \\
y^{f}(k+N-1) \\
y^{s}(k) \\
x(k+N)=A_{c} x(k)+B_{c} U(k) \\
Y(k)=C_{c} x(k)+D_{c} U(k),
\end{array}\right.}
\end{aligned}
$$

$$
\begin{aligned}
A_{c} & =A^{N}, \quad B_{c}=\left[\begin{array}{llll}
A^{N-1} B & A^{N-2} B & \ldots & B
\end{array}\right], \\
C_{c} & =\left[\begin{array}{ccccc}
C^{f^{T}} & A^{T} C^{f^{T}} & \ldots & A^{(N-1)^{T}} C^{f^{T}} & C^{s^{T}}
\end{array}\right]^{T}, \\
D_{c} & =\left[\begin{array}{cccc}
D^{f} & 0 & \ldots & 0 \\
C^{f} B & D^{f} & \ldots & 0 \\
\vdots & \vdots & \ddots & \ldots \\
C^{f} A^{N-2} B & C^{f} A^{N-3} B & \ldots & D^{f} \\
D^{s} & 0 & \ldots & 0
\end{array}\right] .
\end{aligned}
$$

Definition 3.1: The finite zeros of the transfer function $H(z)=C_{c}\left(Z I-A_{c}\right)^{-1} B_{c}+D_{c}$ with minimal realization $\left\{A_{c}, B_{c}, C_{c}, D_{c}\right\}$ are defined to be the finite values of $Z$ for which the rank of the following system matrix falls below its normal rank

$$
Q(Z)=\left[\begin{array}{cc}
Z I-A_{c} & -B_{c} \\
C_{c} & D_{c}
\end{array}\right] .
$$

Moreover, $H(Z)$ is said to have an infinite zero when $n+\operatorname{rank}\left(D_{c}\right)$ is less than the normal rank of $Q(Z)$, or equivalently the rank of $D_{c}$ is less than the normal rank of $H(Z)$.

With the help of Theorem 2.13, we can obtain a result which identifies a class of systems with mixed frequency data which are zero free.

Theorem 3.2: For a generic choice of the matrices $\left\{A, B, C^{s}, C^{f}, D^{s}, D^{f}\right\}$ and $p_{1}>m$, the system matrix of (13) is full column rank for all $Z$.

Proof: With the help of results which we obtained for the single frequency case, the proof is straightforward. Considering the system matrix of $Q(Z)$, a system matrix $Q^{f}(Z)$ can be formed by deleting rows of $Q(Z)$ corresponding to
$C^{s}$. Theorem 2.13 shows that under the condition $p_{1}>m$, $Q^{f}(Z)$ will be of full column rank for all $Z$. Thus, $Q(Z)$ will be of full column rank for all $Z$ and this finishes the proof.

\section{CONCLUSiON}

In this paper, the zero properties of blocked systems resulting from blocking discrete linear systems with mixed frequency data were provided. First, attention was given to the single frequency case, where the unblocked linear systems have all data at the same frequency. In particular, several results were obtained to relate zeros of unblocked linear systems with zeros of their corresponding blocked systems. Then the results for the single frequency case were used to investigate the zero properties of blocked systems resulting from blocking of linear systems with mixed frequency data. It was specifically shown when the blocked systems can be zero free.

\section{REFERENCES}

[1] M. P. Clements and A. B. Galvão, "Macroeconomic forecasting with mixed-frequency data," Journal of Business \& Economic Statistics, vol. 26, pp. 546-554, 2008.

[2] R. Meyer and C. Burrus, "A unified analysis of multirate and periodically time-varying digital filters," Circuits and Systems, IEEE Transactions on, vol. 22, no. 3, pp. 162 - 168, Mar. 1975.

[3] T. Chen and B. A. Francis, Optimal Sampled-Data Control Systems. Secaucus, NJ, USA: Springer-Verlag New York, Inc., 1995.

[4] P. Bolzern, P. Colaneri, and R. Scattolini, "Zeros of discrete-time linear periodic systems," Automatic Control, IEEE Transactions on, vol. 31, no. 11 , pp. 1057 - 1058, Nov. 1986 .

[5] O. M. Grasselli and S. Longhi, "Zeros and poles of linear periodic multivariable discrete-time systems," Circuits, Systems, and Signal Processing, vol. 7, pp. 361-380, 1988.

[6] S. Bittanti, Deterministic and stochastic linear periodic systems, ser. Lecture Notes in Control and Information Sciences. Springer Berlin / Heidelberg, 1986, vol. 86.

[7] S. Bittanti and P. Colaneri, Periodic Systems Filtering and Control, ser. Communications and Control Engineering. Springer-Verlag, 2009.

[8] O. M. Grasselli and S. Longhi, "The geometric approach for linear periodic discrete-time systems," Linear Algebra and its Applications, vol. 158 , pp. $27-60,1991$.

[9] P. Colaneri and S. Longhi, "The realization problem for linear periodic systems," Automatica, vol. 31, no. 5, pp. 775 - 779, 1995.

[10] P. Khargonekar, K. Poolla, and A. Tannenbaum, "Robust control of linear time-invariant plants using periodic compensation," Automatic Control, IEEE Transactions on, vol. 30, no. 11, pp. 1088 - 1096, Nov. 1985.

[11] W. Chen, B. D. O. Anderson, M. Deistler, and A. Filler, "Properties of blocked linear system," in International Federation of Automatic Control Conference, 2011. IFAC'11., 2011.

[12] B. D. O. Anderson and M. Deistler, Properties of Zero-Free Transfer Function Matrices, 2008, vol. 1, no. 4. 